\title{
Quality of Life after Surgical Treatment of Early Barrett's Cancer: A Prospective Comparison of the Ivor-Lewis Resection Versus the Modified Merendino Resection. A Statistical Hint
}

\author{
Alberto Mangano - Georgios D. Lianos • \\ Stefano Rausei - Luigi Boni - Gianlorenzo Dionigi
}

Published online: 3 May 2014

(c) Société Internationale de Chirurgie 2014

Sir,

We read with extreme interest the cutting-edge article published by Zapletal et al. [1] on quality of life (QOL) after surgery for early Barrett's cancer. These colleagues have undoubtedly produced some important enrichments for the scientific literature, and this paper in particular presents new objective results that advance our understanding of esophageal surgery. We would like to offer our additional remarks on this topic.

The authors are to be commended for their efforts in conducting such a prospective study in order to compare the impact on health-related QOL (HR-QOL) of the IvorLewis traditional resection versus the modified Merendino resection [2] in a considerably large sample of patients.

We agree with the authors that, to date, this study is the first to examine the HR-QOL of patients with early esophageal adenocarcinomas 1 and 2 years after surgery. Considering the technical elements of the two surgical techniques, we admit that theoretically a higher QOL for patients treated with the Merendino procedure should be expected. Notably, the Merendino surgical approach, surprisingly, does not seem to show implemented results in terms of QOL.

However, even if the statistical tests used are correct and, as far as we know, the effect to be measured still does not have any validated statistical landmark, it does not seem that the authors have performed a pre hoc sample size calculation. De facto, in every prospective study if statistical tests are used, their power should be determined a priori. While this study is very innovative and gives an objectively conducted comparison of the HR-QOL between the two groups, an accurate power analysis is the only way to draw adequate conclusions in any prospective study. Hence, an extension of this study in a prospective, randomized, well-powered, multi-centered trial could be an interesting idea.

Finally, we would like to thank again the authors for their valuable scientific contribution.

\section{References}

1. Zapletal C, Heesen C, Origer J et al (2013) Quality of life after surgical treatment of early Barrett's cancer: a prospective comparison of the Ivor-Lewis resection versus the modified Merendino resection. World J Surg. doi:10.1007/s00268-0132410-1

2. Gerzic ZB (1997) Modification of the Merendino procedure. Dis Esophagus 4:270-275

\footnotetext{
A. Mangano $(\bowtie) \cdot$ S. Rausei $\cdot$ L. Boni · G. Dionigi Department of Surgical Sciences and Human Morphology, 1st Division of General Surgery, Insubria University, Varese, Italy e-mail: alberto.mangano@gmail.com

G. D. Lianos

Department of Surgery, Ioannina University School of Medicine, Ioannina, Greece
} 